\title{
Soliton compression to few-cycle pulses using quadratic nonlinear photonic crystal fibers: A design study
}

Bache, Morten; Moses, Jeffrey; Lægsgaard, Jesper; Bang, Ole; Wise, Frank

Published in:

CLEO/QELS Technical Digest 2007

Link to article, DOI:

10.1109/CLEO.2007.4452852

Publication date:

2007

Document Version

Publisher's PDF, also known as Version of record

Link back to DTU Orbit

Citation (APA):

Bache, M., Moses, J., Lægsgaard, J., Bang, O., \& Wise, F. (2007). Soliton compression to few-cycle pulses using quadratic nonlinear photonic crystal fibers: A design study. In CLEO/QELS Technical Digest 2007 (pp. CThQ4). IEEE. https://doi.org/10.1109/CLEO.2007.4452852

\section{General rights}

Copyright and moral rights for the publications made accessible in the public portal are retained by the authors and/or other copyright owners and it is a condition of accessing publications that users recognise and abide by the legal requirements associated with these rights.

- Users may download and print one copy of any publication from the public portal for the purpose of private study or research.

- You may not further distribute the material or use it for any profit-making activity or commercial gain

- You may freely distribute the URL identifying the publication in the public portal 


\title{
CThQ4.pdf
}

\section{Soliton Compression to Few-cycle Pulses Using Quadratic Nonlinear Photonic Crystal Fibers: A Design Study}

\author{
Morten Bache ${ }^{1}$, Jeffrey Moses ${ }^{2}$, Jesper Lægsgaard ${ }^{1}$, Ole Bang ${ }^{1}$, and Frank Wise ${ }^{2}$ \\ ${ }^{I}$ COM•DTU Department of Communications, Optics \& Materials, Technical University of Denmark, Bld. 345v, DK-2800 Lyngby, Denmark. \\ ${ }^{2}$ Department of Applied and Engineering Physics, Cornell University, Ithaca, New York 14853. \\ tel: +45 4525 3775, fax: +45 4593 6581, e-mail: $\underline{\text { bache@com.dtu.dk }}$
}

\begin{abstract}
We show theoretically that high-quality soliton compression from $\sim 500$ fs to $\sim 10 \mathrm{fs}$ is possible in poled silica photonic crystal fibers using cascaded $\chi^{(2)}: \chi^{(2)}$ nonlinearities. A moderate group-velocity mismatch optimizes the compression.

(C)2007 Optical Society of America
\end{abstract}

OCIS codes: $320.5520,190.4370,190.5530,320.7140$

Second-harmonic generation (SHG) in the limit of large phase mismatch, given by $\Delta \beta=\beta_{2}-2 \beta_{1}$, induces a Kerr-like nonlinear phase shift on the fundamental wave $(\mathrm{FW})$. Large negative phase shifts can be created, since the phase mismatch determines the sign and magnitude of the effective cubic nonlinearity. This self-defocusing nonlinearity can be used to compress a pulse when combined with normal dispersion, and problems normally encountered due to self-focusing in cubic media are avoided. Thus, having no power limit, in bulk media a self-defocusing soliton compressor can create high-energy near single-cycle fs pulses [1-3]. However, the group-velocity mismatch (GVM) between the FW and second harmonic (SH), as expressed by the inverse group velocity difference $d_{12}=1 / v_{g, 1^{-}}-1 / v_{g, 2}$, limits the pulse quality and compression ratio. Especially very short input pulses ( $<100 \mathrm{fs})$ experience a Raman-like effect with a characteristic time $\tau_{\mathrm{R}, \mathrm{SHG}}=2\left|d_{12}\right| / \Delta \beta[3]$.

Here we address the limits imposed by GVM by using a new medium for cascaded quadratic $\left(\chi^{(2)}: \chi^{(2)}\right)$ soliton compression: thermally-poled silica photonic crystal fibers (PCFs). By poling standard silica fibers strong effective quadratic nonlinearities around $1 \mathrm{pm} / \mathrm{V}$ have been demonstrated [4]. PCFs are interesting because they have very strong wave-guide dispersion that can be tailored: for SHG index-guiding silica PCFs with a triangular holearrangement can have zero GVM for any $\lambda_{1}>780 \mathrm{~nm}$ by adjusting the PCF hole pitch $\Lambda$ and hole diameter $d$ [5]. Our simulations predict that high-quality compression to few-cycle pulses in PCFs is possible. Such a waveguided geometry can extend the compression technique to lower-energy pulses and produce uniformly compressed beams.

The system is modeled by the following coupled quadratic and cubic nonlinear equations for the fields $\mathcal{E}_{j}(z, \tau)$

$$
\begin{gathered}
\hat{L}_{1} \mathcal{E}_{1}+\kappa_{\mathrm{SHG}, 1} \hat{S}_{1} \mathcal{E}_{1}^{*} \mathcal{E}_{2} e^{i \Delta \beta z}+\kappa_{\mathrm{Kerr}, 1} \hat{S}_{1}\left\{\left(1-f_{R}\right) \mathcal{E}_{1}\left(\mathcal{F}_{11}\left|\mathcal{E}_{1}\right|^{2}+2 \mathcal{F}_{12}\left|\mathcal{E}_{2}\right|^{2}\right)+f_{R} \mathcal{E}_{1}(\tau) \int_{-\infty}^{\infty} d s h_{R}(s)\left[\mathcal{F}_{11}\left|\mathcal{E}_{1}(\tau-s)\right|^{2}+\mathcal{F}_{12}\left|\mathcal{E}_{2}(\tau-s)\right|^{2}\right]\right\}=0 \\
\hat{L}_{2} \mathcal{E}_{2}+\kappa_{\mathrm{SHG}, 2} \hat{S}_{2} \mathcal{E}_{1}^{2} e^{-i \Delta \beta z}+\kappa_{\mathrm{Kerr}, 2} \hat{S}_{2}\left\{\left(1-f_{R}\right) \mathcal{E}_{2}\left(\mathcal{F}_{22}\left|\mathcal{E}_{2}\right|^{2}+2 \mathcal{F}_{12}\left|\mathcal{E}_{1}\right|^{2}\right)+f_{R} \mathcal{E}_{2}(\tau) \int_{-\infty}^{\infty} d s h_{R}(s)\left[\mathcal{F}_{22}\left|\mathcal{E}_{2}(\tau-s)\right|^{2}+\mathcal{F}_{12}\left|\mathcal{E}_{1}(\tau-s)\right|^{2}\right]\right\}=0 \\
\kappa_{\mathrm{SHG}, j}=\frac{\omega_{1} d_{\mathrm{eff}}\left|\int_{S} d \mathbf{x}\left[F_{1}^{*}(\mathbf{x})\right]^{2} F_{2}(\mathbf{x})\right|}{c n_{\mathrm{eff}, j} \int d \mathbf{x}\left|F_{j}(\mathbf{x})\right|^{2}}, \quad \kappa_{\text {Kerr }, j}=\frac{\omega_{j} n_{2}}{c \int d \mathbf{x}\left|F_{j}(\mathbf{x})\right|^{2}}, \quad \mathcal{F}_{j k}=\int_{S} d \mathbf{x}\left|F_{j}(\mathbf{x})\right|^{2}\left|F_{k}(\mathbf{x})\right|^{2}, \quad \hat{S}_{j}=1+\frac{i}{\omega_{j}} \frac{\partial}{\partial \tau}
\end{gathered}
$$

where $h_{R}$ is the silica noninstantaneous Raman response, and $F_{j}$ the PCF transverse modes. The propagation and dispersion operators $\hat{L}_{j}$ are extended to the slowly-evolving wave approximation through dispersion corrections, so the equations are valid for single-cycle pulses [6]. The rescaled equations have Kerr and SHG soliton numbers as

$$
N_{\text {Kerr }}^{2}=L_{\mathrm{D}, 1} \frac{P_{1, \mathrm{in}} \omega_{1} n_{2}}{c A_{\mathrm{eff}, 1}^{\mathrm{Kerr}}}, \quad A_{\mathrm{eff}, 1}^{\mathrm{Kerr}}=\frac{\left[\int d \mathbf{x}\left|F_{1}(\mathbf{x})\right|^{2}\right]^{2}}{\int_{S} d \mathbf{x}\left|F_{1}(\mathbf{x})\right|^{4}}, \quad N_{\mathrm{SHG}}^{2}=L_{\mathrm{D}, 1} \frac{P_{1, \mathrm{in}} 2 \omega_{1}^{2} d_{\mathrm{eff}}^{2}}{\varepsilon_{0} c^{3} n_{\mathrm{eff}, 1}^{2} n_{\text {eff }, 2}|\Delta \beta| A_{\text {eff }}^{\mathrm{SHG}}}, \quad A_{\mathrm{eff}}^{\mathrm{SHG}}=\frac{\left[\int d \mathbf{x}\left|F_{1}(\mathbf{x})\right|^{2}\right]^{2} \int d \mathbf{x}\left|F_{2}(\mathbf{x})\right|^{2}}{\left|\int_{S} d \mathbf{x}\left[F_{1}^{*}(\mathbf{x})\right]^{2} F_{2}(\mathbf{x})\right|^{2}}
$$

where $L_{\mathrm{D}, 1}$ is the FW dispersion length, $P_{1, \text { in }}$ the input power, $n_{2}$ the Kerr nonlinear refractive index, $d_{\text {eff }}$ the effective quadratic nonlinearity, $n_{\mathrm{eff}, j}$ the effective PCF mode indices, and " $S$ " indicates integration over the silica region only. It turns out that soliton compression of the $\mathrm{FW}$ is observed only if $N_{\mathrm{SHG}}>N_{\mathrm{Kerr}}$. This poses a constraint on $\Delta \beta$ as

$$
\bar{N}^{2}=\frac{N_{\mathrm{SHG}}^{2}}{N_{\text {Kerr }}^{2}}=\frac{2 \omega_{1} d_{\mathrm{eff}}^{2} A_{\mathrm{eff}, 1}^{\mathrm{Ker}}}{\varepsilon_{0} c^{2} n_{\mathrm{eff}, 1}^{2} n_{\mathrm{eff}, 2} n_{2}|\Delta \beta| A_{\mathrm{eff}}^{\mathrm{SHG}}}>1 \Rightarrow|\Delta \beta|<\Delta \beta_{c}=\frac{\omega_{1} d_{\text {eff }}^{2} A_{\mathrm{eff}, 1}^{\mathrm{Kerr}}}{c n_{\mathrm{eff}, 1} n_{\mathrm{eff}, 2} n_{2} A_{\mathrm{eff}}^{\mathrm{SHG}}}
$$

Besides this requirement, a lower limit is imposed by GVM: the nonlinear phase shift has to be built up before GVM separates the FW and SH fields [1]. This so-called stationary regime is obtained when $\Delta \beta>>\pi \mid d_{12} / / T_{1, \text { in }}[1]$. We also recall that the nonlinear phase shift is only induced in the cascading limit where the phase mismatch is large. Together with the above requirements this opens a "window" of the phase mismatch for observing compression. It is clear that $d_{\text {eff }}$ must be large compared to $n_{2}$, and similarly the quadratic effective mode area $A_{\text {eff }}^{\mathrm{SHG}}$ must be small compared to the cubic $A_{\text {eff, } 1}^{\text {Kerr }}$ in order to "open" the window.

Based on the results of Ref. [5], Table 1 shows some PCF compressor designs found for $\lambda_{1}=1060 \mathrm{~nm}$ with small or zero GVM. It was not a trivial task, since most designs had anomalous dispersion. Moreover, when GVM is reduced the phase mismatch is increased substantially (typically $\Delta \beta>100 \pi / \mathrm{mm}$ ). Thus, large $d_{\text {eff-values }}(3-5 \mathrm{pm} / \mathrm{V}$ ) had to be used in order to increase $\Delta \beta_{c}$ beyond $\Delta \beta$; these values are probably unrealistic in poled silica fibers. When 


\section{CThQ4.pdf}

using a more modest $d_{\mathrm{eff}}=1 \mathrm{pm} / \mathrm{V}, \Delta \beta_{c}$ becomes smaller than $\Delta \beta$. A solution is to use a slightly mismatched quasiphase matched (QPM) grating that effectively reduces $\Delta \beta$ below $\Delta \beta_{c}$, but still keeping it in the cascading limit. Numerical simulations were used to determine the influence of reducing GVM from large, case (3), to zero, case (1).

Table 1. Selected PCF compressor designs using a hole pitch $\Lambda=0.9 \mu \mathrm{m}$. ${ }^{\dagger}$ Only for Fig. 1(a)-(b). ${ }^{\ddagger}$ Only for Fig. 1(c).

\begin{tabular}{|c||c|c|c|c|c|c|c|c|c|c|c|c|}
\hline Case & $\begin{array}{c}d \\
\mu \mathrm{m}\end{array}$ & $\begin{array}{c}\Delta \beta \\
\mathrm{mm}^{-1}\end{array}$ & $\begin{array}{c}d_{12} \\
\mathrm{fs} / \mathrm{mm}\end{array}$ & $\begin{array}{c}\beta_{21} \\
\mathrm{fs}^{2} / \mathrm{mm}\end{array}$ & $\begin{array}{c}\beta_{22} \\
\mathrm{fs}^{2} / \mathrm{mm}\end{array}$ & $\begin{array}{c}P_{1, \text { in }} \\
\mathrm{W}\end{array}$ & $\begin{array}{c}E_{1, \text { in }} \\
\mathrm{pJ}\end{array}$ & $\begin{array}{c}d_{\text {eff }}^{\dagger} \\
\mathrm{pm} / \mathrm{V}\end{array}$ & $\begin{array}{c}\tau_{R, \mathrm{SHG}} \\
\mathrm{fs}\end{array}$ & $\begin{array}{c}\Lambda_{\mathrm{QPM}} \\
\mu \mathrm{m}\end{array}$ & $\begin{array}{c}\Delta \beta_{\mathrm{eff}} \\
\mathrm{mm}^{-1}\end{array}$ & $\begin{array}{c}\tau_{R, \mathrm{SHG}} \\
\mathrm{fs}\end{array}$ \\
\hline \hline$(1)$ & 0.63 & 721 & 0 & 41 & 34 & 500 & 227 & 5.5 & 0.0 & 8.83 & 9.71 & 0.0 \\
\hline$(2)$ & 0.54 & 596 & -33 & 87 & 40 & 1290 & 585 & 5.0 & 0.1 & 10.71 & 9.63 & 6.8 \\
\hline$(3)$ & 0.45 & 474 & -72 & 138 & 46 & 3000 & 1362 & 4.5 & 0.3 & 13.54 & 9.52 & 14.8 \\
\hline
\end{tabular}

We first tried a large $d_{\mathrm{eff}}=4.5-5.5 \mathrm{pm} / \mathrm{V}$ and no QPM grating. The effect of reducing GVM turned out to be less important than expected: we were able to observe strong compression from 100-800 fs pulses to 10-20 fs pulses even with GVM present. The result of the three cases of Table 1 is shown in Fig. 1(a)-(b), and actually case (2) and (3), where GVM is present, provide a slightly cleaner and more compressed pulse than case (1). The explanation can be found by observing the corresponding SH pulses in Fig. 1(b): for zero GVM, case (1), the SH has oscillations that distort the final FW pulse, which are caused by the intensity-dependence of the conversion/back-conversion cycle of the waves. In the nonzero GVM cases (2) and (3) they are quenched since the GVM continuously transfers energy away from the pulse center. The pulse qualities are all very good because $\Delta \beta$ was so large that the characteristic times of the Raman-like perturbation $\tau_{\mathrm{R}, \mathrm{SHG}}=2\left|d_{12}\right| / \Delta \beta$ (see Table 1) are much less than that of silica $\left(\tau_{\mathrm{R}} \sim 3 \mathrm{fs}\right.$ ).

While the above results are good, they are obtained for $d_{\mathrm{eff}}>4 \mathrm{pm} / \mathrm{V}$. We show in Fig. 1(c) that for a more realistic value $d_{\mathrm{eff}}=1 \mathrm{pm} / \mathrm{V}$ equally good compression is achieved by using a slightly mismatched QPM grating having a pitch $\Lambda_{\mathrm{QPM}}$. Fig. 1 (c) shows that in case (1) with zero GVM a similar pulse is observed as with large $d_{\mathrm{eff}}$ and no QPM grating, while case (3) has very poor compression and quality. Case (2) has instead medium GVM, and gives the best pulse quality. This is again related to the removal of the SH oscillations in the pulse center. Thus, when using a QPM grating it was very important that GVM was not too large. This is because the grating gave an effective phase mismatch $\Delta \beta_{\text {eff }}=\Delta \beta-2 \pi / \Lambda_{\mathrm{OPM}}$ that was much smaller, implying that the characteristic time of the Raman-like perturbation due to GVM $\tau_{\mathrm{R}, \mathrm{SHG}, \mathrm{eff}}=2\left|d_{12}\right| / \Delta \beta_{\text {eff }}$ became much larger than that of silica (see Table 1). Such a large $\tau_{\mathrm{R}, \mathrm{SHG} \text {,eff }}$ results in the asymmetric shape and pulse splitting observed in Fig. 1(c) case (3). Finally the SH conversion was quite strong because the process is almost phase matched (1-10\% conversion of the input energy).
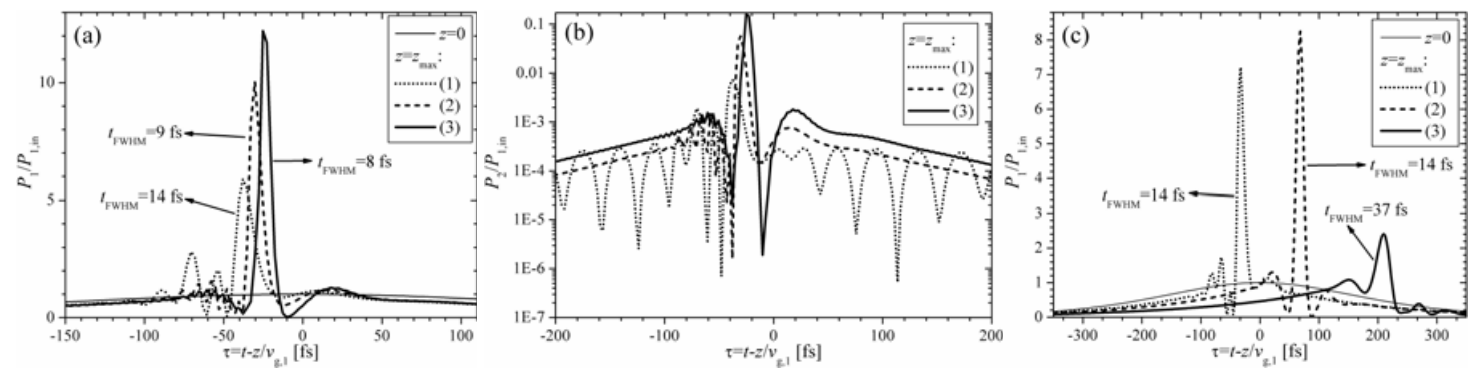

Fig. 1. Compression of a 400 fs pulse for $N_{\mathrm{SHG}}=14.6$ and $N_{\mathrm{Kerr}}=8.2$. (a) FW and (b) SH power with $d_{\mathrm{eff}}$ large and no QPM grating. (c) FW power using $d_{\mathrm{eff}}=1 \mathrm{pm} / \mathrm{V}$ and a slightly mismatched QPM grating. $n_{2}=2.2 \cdot 10^{-20} \mathrm{~m}^{2} / \mathrm{W} . z_{\max }$ is the maximum compression point (varies from case to case).

In conclusion we found that the competition between the quadratic and cubic nonlinearities required quite large quadratic nonlinearities from thermal poling of the silica PCF in order to be able to observe pulse compression. Achieving the necessary design is within practical reason, however, especially when imposing a slightly mismatched QPM grating. Compression ratios as high as $\sim 50$ to few-cycle pulses at $\lambda_{1}=1060 \mathrm{~nm}$ were demonstrated theoretically, and good pulse qualities were observed when the GVM was small due to a reduced Raman-like perturbation. The best pulse quality was surprisingly not obtained with zero GVM, but rather with a small GVM.

[1] X. Liu, L. Qian, and F. W. Wise, "High-energy pulse compression by use of negative phase shifts produced by the cascaded $\chi^{(2)}: \chi^{(2)}$ nonlinearity," Opt. Lett. 24, 1777-1779 (1999).

[2] S. Ashihara et al., "Soliton compression of femtosecond pulses in quadratic media," J. Opt. Soc. Am. B 19, 2505-2510 (2002).

[3] J. Moses and F. W. Wise, "Soliton compression in quadratic media: high-energy few-cycle pulses with a frequency-doubling crystal," Opt. Lett. 31, 1881-1883 (2006).

[4] P. G. Kazansky and V. Pruneri, "Electric-field poling of quasi-phase-matched optical fibers," J. Opt. Soc. Am. B 14, $3170-3179$ (1997).

[5] M. Bache et al., "Tuning quadratic nonlinear photonic crystal fibers for zero group-velocity mismatch," Opt. Lett. 31, 1612-1614, (2006).

[6] J. Moses and F. W. Wise, "Controllable Self-Steepening of Ultrashort Pulses in Quadratic Nonlinear Media," Phys. Rev. Lett. 97, 073903 (2006), see also arXiv:physics/0604170. 